\title{
ORIGINAL ARTICLE \\ The use of the neurologic exam to predict awareness and control of lower urinary tract function post $\mathrm{SCl}$
}

\author{
MS Alexander ${ }^{1,2,3}$, C Carr ${ }^{2}$, Y Chen ${ }^{2}$ and A McLain ${ }^{2}$
}

Study Design: Cross-sectional study.

Objectives: To assess whether T11-L2 sensation is significantly associated with perception of bladder filling and whether S3-S5 sensation is significantly associated with potential for voluntary voiding after spinal cord injury (SCl).

Setting: Alabama, United States.

Methods: A total of 79 men and women with SCl, and 40 able-bodied (AB) subjects, ages 19-60, seen in an outpatient academic medical center clinic, underwent International Standards for Neurological Classification of SCI (ISNCSCI) assessment along with urodynamic testing to assess lower urinary tract (LUT) function. $X^{2}$ test was performed to compare: (1) bladder sensation across T11-L2 groups classified by total sensory scores and (2) ability to voluntarily void across S3-S5 scores.

Results: Persons with greater ability to perceive pinprick and light touch sensation in the T11-L2 dermatomes were more likely to perceive bladder filling both via self-report and by urodynamics. However, persons with greater sensation at T6-T9 and S3-5 also had a greater likelihood of perceiving bladder filling. Subjects with greater preservation of sensation in S3-5 reported greater ability to initiate and control voiding.

Conclusion: Findings suggest ISNCSCI results along with self-report can be used to predict potential for bladder control.

Sponsorship: National Institute of Disability and Rehabilitation Research.

Spinal Cord (2017) 55, 840-843; doi:10.1038/sc.2017.55; published online 30 May 2017

\section{INTRODUCTION}

Neurogenic bladder is a common problem for persons with spinal cord injuries (SCIs) and maintenance of continence often requires unsightly, costly appliances. Therapies are available to improve motor function for activities of daily living and ambulation; however, limited attention is focused on improving voluntary control of voiding. Moreover, we do not have simple bedside tools to predict the recovery of volitional control of LUT functions.

Bladder, bowel and sexual function rely on the thoracolumbar sympathetic responses via the hypogastric nerves, sacral parasympathetic responses via the pelvic nerves and somatic sacral responses via the pudendal nerve. Information is available to predict the impact of specific levels and types of SCI on recovery of sexual function ${ }^{1-3}$ and a recent report described a formula to predict urinary continence and complete bladder emptying 1 year after SCI. ${ }^{4}$ This formula relies on lower extremity motor score, S3 light touch sensation and spinal cord independence measure (SCIM) subscales for sphincter management and respiration and this report documents persons who have complete continence at 40 days are likely to maintain it at 1 year. Physicians can rely on their determination of sacral reflexes to document the type of bladder dysfunction; however, many physicians do not perform anal reflexes. Moreover, little information is available to predict which patients with partial control have potential to regain LUT volitional control.
Urodynamics is considered the gold standard for the evaluation of LUT function, despite the fact that many questions exist as to the optimal way to describe and measure bladder sensation. ${ }^{5}$ Urodynamics has been used to study sensation of bladder fullness and the ability to control the timing of voiding in the able-bodied; ${ }^{6-8}$ however, many people do not have access to this technology. Because of this, a more basic way to measure sensation may be useful and other reports ${ }^{9-11}$ have examined levels of SCI and whether preservation of hypogastric nerve innervation contributes to the ability to perceive sensation of bladder filling. Additionally, S4-S5 pinprick sensation at $72 \mathrm{~h}$ post $\mathrm{SCI}^{12}$ combined with retention of position sense has been correlated with the natural ability to void.

We have previously shown ${ }^{2,3}$ the combined sensory score for pinprick and light touch sensation in the T11-L2 dermatomes could be used to predict the capacity for women and men with SCIs to experience psychogenic genital vasocongestion. In addition, we showed the ability to experience orgasm was significantly diminished in those subjects with complete LMN injuries affecting the S3-S5 cord segments as compared to subjects with all other levels and degrees of SCIs. ${ }^{1,2}$ As neurologic control of both sexual and bladder function is similar, we hypothesized retention of the ability to perceive sensation in the T11-L2 dermatomes would be significantly related to the ability to experience some type of sensation associated with bladder filling. We also hypothesized the degree of preserved sensation at S3-S5 could be used to determine the potential for voluntary voiding.

${ }^{1}$ Physical Medicine and Rehabilitation, Birmingham VA Medical Center, Birmingham, AL, USA; ${ }^{2}$ Department of Physical Medicine and Rehabilitation, University of Alabama School of Medicine, Birmingham, AL, USA and ${ }^{3}$ Department of Physical Medicine and Rehabilitation, Harvard University School of Medicine, Boston, MA, USA Correspondence: Dr MS Alexander, Physical Medicine and Rehabilitation, Birmingham VA Medical Center, 700 S. 19th Street, Birmingham, AL 35233, USA. E-mail: spinalcordmd@live.com

Received 2 November 2016; revised 18 April 2017; accepted 24 April 2017; published online 30 May 2017 
Table 1 Injury levels

\begin{tabular}{|c|c|}
\hline Level of injury & $N=79$ \\
\hline $\mathrm{C} 1$ & 1 \\
\hline $\mathrm{C} 2$ & 3 \\
\hline $\mathrm{C} 4$ & 6 \\
\hline C5 & 11 \\
\hline $\mathrm{C} 6$ & 11 \\
\hline C7 & 3 \\
\hline $\mathrm{C} 8$ & 1 \\
\hline $\mathrm{T} 1$ & 1 \\
\hline $\mathrm{T} 2$ & 1 \\
\hline T3 & 6 \\
\hline $\mathrm{T} 4$ & 1 \\
\hline T5 & 2 \\
\hline T6 & 1 \\
\hline $\mathrm{T} 7$ & 2 \\
\hline T8 & 2 \\
\hline T9 & 1 \\
\hline T10 & 8 \\
\hline T11 & 5 \\
\hline $\mathrm{T} 12$ & 2 \\
\hline L1 & 1 \\
\hline L2 & 7 \\
\hline L3 & 1 \\
\hline L4 & 2 \\
\hline
\end{tabular}

\section{METHODS}

\section{Subjects}

Inclusion criteria were ages 19-60, and over 6 months post traumatic SCI. Exclusion criteria were prior traumatic brain injury or other disorder that could affect neurologic or LUT function. In order to obtain as natural a result as possible regarding bladder filling volumes, subjects on medications affecting bladder function were required to discontinue these medications for $72 \mathrm{~h}$ prior to the study. Postmenopausal women were required to be on vaginal or oral estrogens.

\section{Methods}

The study was approved by the Institutional Review Board of the University of Alabama at Birmingham. All subjects provided informed consent. History and physical examination was performed, including detailed ISNCSCI exam ${ }^{13}$ with special attention to the T11-L2 and S3-S5 areas in addition to the genital examination, which included assessment of anal sensation, the ability to voluntarily contract the rectal sphincter, assessment of the presence of the bulbocavernosus reflex, anal wink reflex and cremasteric reflex.

Prior to performance of urodynamic studies, a urine specimen was obtained and if the urine was infected, culture and sensitivity was performed with urodynamic testing rescheduled a week later. If appropriate, patients were treated for 7 days with antibiotics. All evaluations were thus performed on 'clean' bladders. For documentation of bladder sensation and pressure, we used the International Continence Society (ICS) ${ }^{14}$ guidelines and performed cystometry with simultaneous imaging for observation of the morphology and function of the LUT. Bladder sensation, including volume at first sensation to void, normal desire to void, strong desire to void, urgency and maximum cystometric capacity were monitored as were detrusor overactivity, leak and compliance. On the basis of these results, the urologist reported the sensation of bladder filling as normal, impaired or absent.

In subjects at the level of T6 and above, routine procedures were performed for autonomic dysreflexia prophylaxis. These included performance of blood pressure (BP) before and every $5 \mathrm{~min}$ during the procedure and continuing every 5 min until stable post procedure. In addition, a protocol for treatment of potential dysreflexia was included.

\section{Statistical analyses}

$X^{2}$ test was performed to compare bladder sensation across T11-L2, T6-T9 and S3-S5 groups. At T11-L2 and T6-T9 sensory scores were broken up into $0-8,9-23$ and $24-32$, and $A B$ individuals. The ability to voluntarily void across S3-S5 scores (0,1-12, 13-16 and AB individuals) was also assessed by the chi-square method.

\section{RESULTS}

Subjects

Subjects included 85 persons with SCIs along with 40 able-bodied subjects. Of these, six persons with SCIs were excluded from the final sample due to incomplete urodynamic data. Of the final sample there were 44 males and 35 females with SCIs and 20 males and 20 females that were able-bodied. Persons with SCIs were a mean age of 35.9 with a range of 20-59 and able-bodied subjects were 33.5 with a range of 19-64. Subjects with SCIs were a mean of $4.39+/-4.38$ years post injury. Of subjects with SCIs, 36 had cervical lesions, 32 had thoracic lesions and 11 had lumbar lesions. Frequency of specific injury levels is listed in Table 1. With regards to completeness of injury 33 subjects (42\%) were classified as AIS A, 5 (6\%) were AIS B, 15 (19\%) were AIS C, $21(27 \%)$ were AIS D and $5(6 \%)$ were AIS E. Method of bladder management is listed in Table 2. There were no statistically significant differences in age, race, years of education or marital status between SCI and $\mathrm{AB}$ groups.

\section{Preservation of sensation of bladder filling}

We first performed an analysis of all SCI subjects to look at the overall correlation between self-report of bladder filling and sensation of bladder filling based upon urodynamics measurement. The categories increased, altered and decreased were modified to reduced and possible choices include absent, reduced, or normal. Results revealed moderate agreement $(\kappa=0.62)$ for this measure. Next we analyzed subgroups. All able-bodied subjects had normal bladder sensation via both self-report and urodynamics. SCI subjects were divided based upon total sensory scores at T11-L2 to pinprick and light touch. On the basis oof this grouping, there were 36 SCI subjects with $0-8$ sensory scores, 19 with $9-23$ and 24 with $24-32$. There was a significant difference between groups with regards to the presence of bladder sensation both by urodynamics $(P=0.002)$ and verbally $(P<0.0001)$ with the more sensation subjects had preserved at T11-L2, the more likely they were to have sensation of bladder filling (Table 3). The subgroup of subjects with no sensation between S3-S5 $(n=43)$ was also compared with regards to filling sensation during urodynamics. On the basis of grouping at T11-L2, there was not a statistically significant difference between groups $(P=0.33)$.

Subjects were grouped based upon sensory scores at S3-S5 versus bladder filling sensation. There were 43 subjects with 0,18 with 1-12 and 18 with scores of 13-16. There was a significant difference between groups with regards to sensation of filling via urodynamics $(P=0.0003)$ and via self-report $(P<.001)$. We then compared the subjects with a total sensory score of $24-32$ at T11-L2 $(n=24)$ to see if there was a difference in preservation of filling sensation based upon sacral sensation. In this subpopulation, there was not a statistically significant difference between groups $(P=0.31)$. Subjects were also divided based upon sensation at T6-T9 and compared to see their ability to appreciate sensation of bladder filling. In this subpopulation, there were 22 subjects with 0-8 sensation, 14 with 9-23 and 43 with 24-32 sensory scores. Utilizing this grouping there was a significant difference between groups with regards to both sensation of bladder filling via urodynamics $(P=.01)$ and via self-report $(P=0.0006)$. In a further assessment, we looked at subjects whose sensory score at 
Table 2 Method of bladder management

\begin{tabular}{lcc}
\hline Method & Number & Percentage \\
\hline Normal & 27 & 34 \\
Intermittent catheterization & 26 & 33 \\
Reflex voiding/condom catheter & 12 & 15 \\
Foley & 10 & 13 \\
Continent diversion & 2 & 3 \\
Manual expression & 2 & 3 \\
\hline
\end{tabular}

Table 3 Preservation of sensation of bladder filling across T11-L2 groups

\begin{tabular}{lcccc}
\hline & \multicolumn{3}{c}{ T11-L2 total sensory score } & \\
\cline { 2 - 4 } & $0-8$ & $9-23$ & $24-32$ & \\
\hline Sample size & 36 & 19 & 24 & \\
& & & \\
Urodynamic study: bladder filling sensation, $\mathrm{n}(\%)$ & & \\
Absent & $15(41.7)$ & $3(15.8)$ & $1(4.2)$ & \\
Partial & $18(50.0)$ & $10(52.6)$ & $12(50.0)$ & \\
Normal & $3(8.3)$ & $6(31.6)$ & $11(45.8)$ & \\
& & & \\
Self-reported sensation of need to empty bladder, $\mathrm{n}(\%)$ & \\
Absent & $17(47.2)$ & $1(5.3)$ & $1(4.2)$ & \\
Reduced & $16(44.4)$ & $13(68.4)$ & $7(29.2)$ & \\
Normal & $3(8.3)$ & $5(26.3)$ & $16(66.7)$ & \\
\hline
\end{tabular}

T11-L2 was 0-8 $(n=36)$ and then compared their sensory scores at T6-T9 to see if there was a significant difference between groups looking at bladder sensation during urodynamics. There was not a statistically significant difference between groups $(P=0.62)$.

\section{Sacral sensation and voluntary control of voiding}

On the basis of the above groupings, the preservation of sacral sensation at S3-S5 was compared with ability to voluntarily initiate voiding (Table 4). Subjects with greater preservation of sensation were more likely to retain the ability to voluntarily initiate voiding $(P<.0001)$. Additionally, subjects also reported greater preservation of voluntary voiding control based upon the presence of sensation in this area $(P<.0001)$.

\section{DISCUSSION}

One goal of this research was to determine if testing sensory function at the T11-L2 dermatomes would be helpful to predict an individual with SCI's ability to perceive sensation of bladder filling both via urodynamics and self-report. We found an association between the preservation of sensory function in T11-L2 and the ability to perceive sensation of bladder filling via both methods. However, there was also an association between perceived sensation of bladder filling via self-report and urodynamics based upon preservation of sensation at either T6-T9 or S3-5. Therefore, results only partially supported our initial hypothesis. We hypothesize that sympathetic neuroplasticity is responsible for this discrepancy. Formation of new sympathetic connections likely resulted in the association we observed between greater sensory scores at areas adjacent to T11-L2 despite minimal retention of surface sensation in the T11-L2 dermatomes. ${ }^{15}$ Other possibilities are the inaccuracy of the examinations or an insufficient $\mathrm{N}$ to obtain statistically significant results.
Table 4 Self-reported voluntary control of voiding across S3-S5 group

\begin{tabular}{|c|c|c|c|c|}
\hline & \multicolumn{3}{|c|}{ S3-S5 total sensory score } & \multirow[t]{2}{*}{$P$} \\
\hline & 0 & $1-12$ & $13-16$ & \\
\hline Sample size & 43 & 18 & 18 & \\
\hline \multicolumn{5}{|c|}{ Voluntary voiding initiation, n (\%) } \\
\hline Absent & $32(74.4)$ & $7(38.9)$ & $1(5.6)$ & $<0.0001$ \\
\hline Reduced & $7(16.3)$ & $8(44.4)$ & $5(27.8)$ & \\
\hline Normal & $4(9.3)$ & $3(16.7)$ & $12(66.7)$ & \\
\hline \multicolumn{5}{|c|}{ Voluntary voiding control, n (\%) } \\
\hline Absent & $31(72.1)$ & $6(33.3)$ & $0(0.0)$ & $<0.0001$ \\
\hline Reduced & $10(23.3)$ & $11(61.1)$ & $8(44.4)$ & \\
\hline Normal & $2(4.7)$ & $1(5.6)$ & $10(55.6)$ & \\
\hline
\end{tabular}

Our second goal was to determine if the preservation of S3-S5 sensation would be predictive of the ability to initiate and control voluntary voiding. In this area, there was a statistically significant association for both functions, thus, retention of voluntary control of bladder function should be attempted with persons with preservation of greater sensory function between S3 and S5.

Although these results are limited and we did not obtain definitive documentation of the best dermatomes to use to predict LUT responses, we believe they highlight the need for further study. Detailed assessment of the sensory function in these dermatomes is already available in many persons with SCI and it should be possible to correlate the retention of sensation with the recording of bladder function. Due to a lack of consensus of what normal bladder volumes are for various filling sensations, we did not use the actual urodynamic data, rather we used the urologist's report of patients bladder sensation during urodynamics, based upon the International Standards to Document Remaining Autonomic Function after SCI (ISAFSCI) $)^{16}$ recommendations.

Our study complements the previous studies predicting volitional control of voiding as these studies were directed more at predicting recovery of normal urinary neurologic function, rather than seeking ways to find partial preservation of volitional urinary tract control after SCI. Current clinical practice does not place significant emphasis on maximizing recovery of voluntary control of the bladder. By allowing clinicians to be able to better predict specific aspects of autonomic function with a simple neurologic exam, targeted methods to retrain neurogenic bladders should be facilitated. ${ }^{17}$ For instance, a person with SCI and greater preservation of sensory function between T6 and L2 or S3-S5 should also have more reliable sensation of bladder filling. Thus, we can consider using this information to encourage patients with greater remaining surface sensation to catheterize themselves based on their sensations related to bladder filling in addition to timed catheterizations, provided this is done with attention to bladder filling pressures and safe voiding. In addition, because voluntary voiding should be possible in persons with preservation of sensory function at S3-S5 spinal segments, we may be able to develop improved methods to hold off the urge to void or voluntarily initiate voiding in persons with greater retained sensation. Improving either of these functions has potential to be cost-effective since they place emphasis on optimizing the patient's natural neurologic potential. Moreover, these techniques may also be beneficial to consider for practitioners working 
with patients with SCIs in developing countries. Our findings would be more powerful if the data were obtained prospectively and correlated with ongoing detailed assessment using the ISAFSCI, International Lower Urinary Tract Function Basic Spinal Cord Injury Data Set ${ }^{18}$ and the International Urodynamic Basic Spinal Cord Injury Data Set. ${ }^{19}$ Our study provides more detailed self-report information about control of voiding as we consider both the ability to initiate voiding and prevent leakage, which is not done in the other documents. Ideally, we recommend that this information be added to future versions of the ISAFSCI. Moreover, all three documents should be used together because the ISAFSCI is the only that seeks to identify subjects with partial control of LUT function who may have potential for greater recovery of function than those persons with absent function. A prospective study with this data would also be useful to understand the natural history of recovery of voluntary bladder function after SCI and assist in prediction of which persons with SCIs would most likely benefit from experimental therapies designed to promote neurologic and autonomic recovery.

\section{CONCLUSION}

In this study, we assessed the association of remaining surface sensation after SCI with sensation of bladder filling and control of voluntary voiding. Use of the ISNCSCI coupled with the ISAFSCI may provide clinical ways to determine those patients with greater potential for recovery of voiding without appliances. Further research is warranted to study these associations.

\section{DATA ARCHIVING}

There were no data to deposit.

\section{CONFLICT OF INTEREST}

The authors declare no conflict of interest.

\section{ACKNOWLEDGEMENTS}

This work was supported by the United States National Institute of Disability, Independent Living, and Rehabilitation Research.
1 Sipski M, Alexander CJ, Gomez-Marin O. Effects of level and degree of spinal cord injury on male orgasm. Spinal Cord 2006; 44: 798-804.

2 Sipski ML, Alexander CJ, Rosen RC. Sexual arousal and orgasm in women: effects of spinal cord injury. Ann Neurol 2001; 49: 35-44.

3 Sipski ML, Alexander CJ, Gomez O, Spalding J. The effects of spinal cord injury on psychogenic sexual arousal in males. J Urol 2007; 177: 247-251.

4 Pavese C, Schneider MP, Schubert M, Curt A, Sciovoletto G. Finazzi-Agro E et al. Prediction of bladder outcomes after traumatic spinal cord injury: A longitudinal cohort study. PLoS Med 2016; 13: e1002041.

5 De Wachter S, Smith P, Tannenbaum C, Van Koevering G, Drake M, Wyndale JJ et al. How should bladder sensation be measured?: ICI-RS 2011 Neurourol. Urodynamic 2012; 31: 370-374.

6 Wyndaele JJ. The normal pattern of perception of bladder filling during cystometry studied in 38 young healthy volunteers. J Urol 1998; 160: 479-481.

7 Wyndaele JJ. Cystometrical sensory data from a normal population: comparison of two groups of young healthy volunteers examined with 5 years interval. Eur Urol 2002; 42: 34-38.

8 DeWachter S, Wyndaele JJ. Does bladder tone influence sensation of filling and electrosensation in the bladder? A blind controlled study in young health volunteers using bethanechol. J Urol 2001; 165: 802-804.

9 Wyndaele JJ. Investigation of the afferent nerves of the lower urinary tract in patient with "complete" and "incomplete" spinal cord injury. Paraplegia 1991; 29: 490-494.

10 Wyndaele JJ. Correlation between clinical neurological data and urodynamic function in spinal cord injured patients. Spinal Cord 1997; 35: 213-216.

11 Ersos M, Akyuz M. Bladder-filling sensation in patients with spinal cord injury and the potential for sensation-dependent bladder emptying. Spinal Cord 2004; 42: 110-116.

12 Weiss DJ, Fried GW, Chancellor MB, Herbison GH, Ditunno JF, Staas WE. Spinal cord injury and bladder recovery. Arch Phys Med Rehabil 1996; 77: 1133-1135.

13 American Spinal Injury Association. International Standards for Neurological Classification of Spinal Cord Injury, Revised 2000. American Spinal Injury Association: Chicago, IL, USA. 2000.

14 Schäfer W, Abrams P, Liao L, Mattiasson A, Pesce F, Spangberg A et al. Good urodynamic practices: uroflowmetry, filling cystometry, and pressure-flow studies. Neurourol Urodyn 2002; 21: 261-274.

15 Mclachlan EM. Diversity of sympathetic vasoconstrictor pathways and their plasticity after spinal cord injury. Clin Auton Res 2007; 17: 6-12.

16 Alexander MS, Biering-Sorensen F, Bodner D, Brackett NL, Cardenas D, Charlifue S et al. International standards to document remaining autonomic function after spinal cord injury. Spinal Cord 2009; 47: 36-43.

17 Vásquez N, Knight SL, Susser J, Gall A, Ellaway PH, Craggs M. Pelvic floor muscle training in spinal cord injury and its impact on neurogenic detrusor over-activity and incontinence. Spinal Cord 2015; 53: 887-889.

18 Biering-Sorensen F, Craggs M, Kennelly M, Schick E, Wyndaele J-J. International lower urinary tract function basic spinal cord injury data set. Spinal Cord 2008; 46 . 325-330.

19 Biering-Sorensen F, Craggs M, Kennelly M, Schick E, Wyndaele J-J. International urodynamic basic spinal cord injury data set. Spinal Cord 2008; 46: 513-516. 